\title{
Quand c'est la science qui bricole, c'est du sérieux
}

Bricolage d'objets et d'images dans le domaine des neurosciences

\section{Giulia Anichini}

\section{(2) OpenEdition}

Journals

Édition électronique

URL : https://journals.openedition.org/tc/7305

DOI : $10.4000 /$ tc. 7305

ISSN : 1952-420X

Éditeur

Éditions de l'EHESS

\section{Édition imprimée}

Date de publication : 15 décembre 2013

Pagination : 212-235

ISBN : 978-2-7351-1654-6

ISSN : 0248-6016

Référence électronique

Giulia Anichini, «Quand c'est la science qui bricole, c'est du sérieux», Techniques \& Culture [En ligne], 61 | 2013, mis en ligne le 15 décembre 2016, consulté le 29 septembre 2022. URL : http:// journals.openedition.org/tc/7305; DOI : https://doi.org/10.4000/tc.7305 


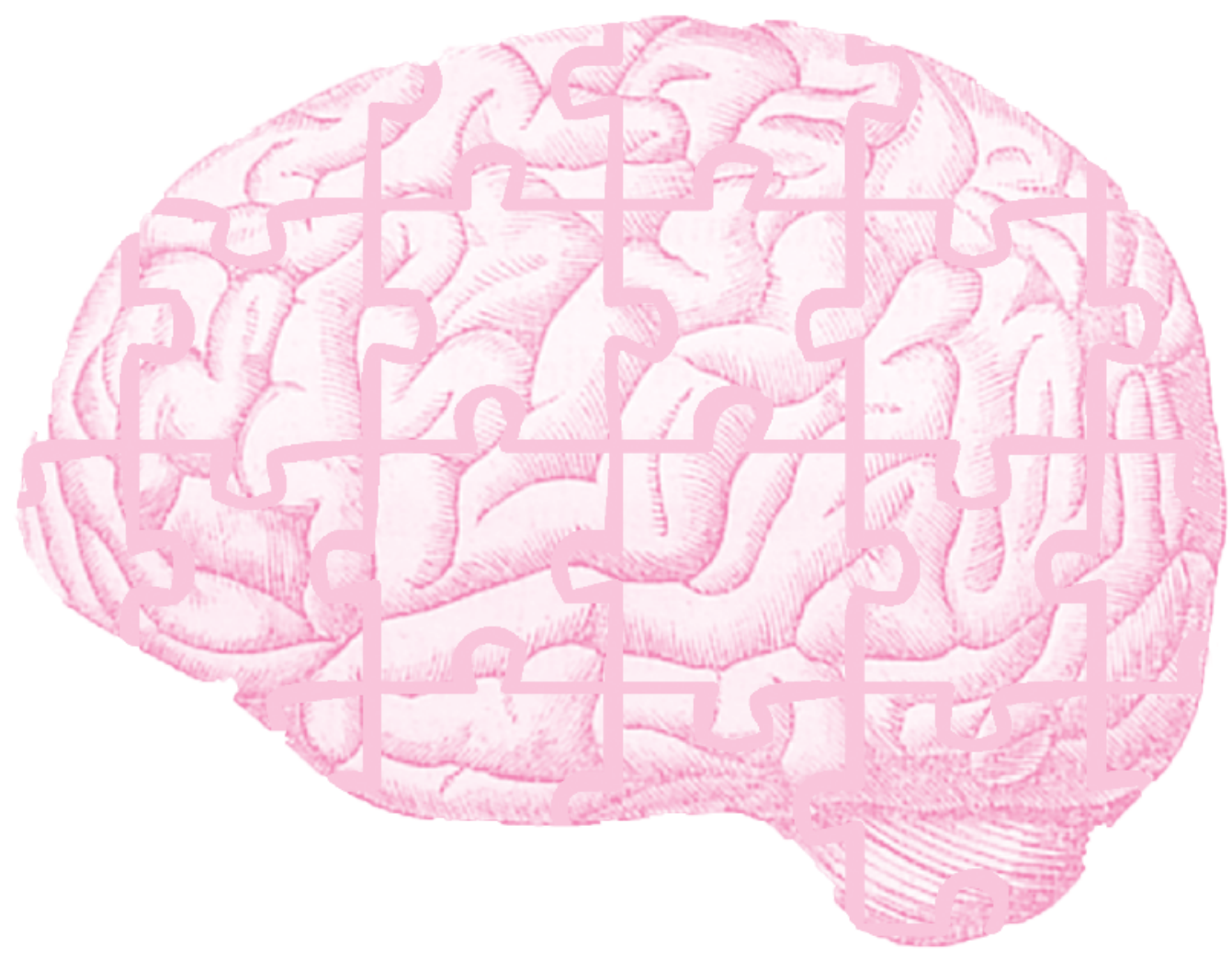

엉 


\section{QUAND C'EST LA SCIENCE QUII BRICOLE, C'EST DU SÉRIEUX}

\section{Bricolage d'objets et d'images dans le domaine des neurosciences}

Le premier objectif de cet article est de comparer les différentes déclinaisons du mot «bricolage» dans des analyses sociologiques du travail scientifique. Celles-ci témoignent de la diversité d'exigences auxquelles ce concept répond, de la variabilité de son influence sur la production des connaissances, de sa valeur contextuelle. Comme on le verra, le bricolage désigne tout aussi bien des ajustements contingents qui limitent leur champ d'action à l'accomplissement d'une tâche que l'agencement stratégique des données. Les deux pratiques ne répondent pas aux mêmes contraintes et sont différemment conçues. Cela amène au deuxième objectif, celui de proposer une classification des pratiques de bricolage, en s'appuyant sur la littérature existante et sur des exemples ethnographiques. Ces derniers nous donnent à voir dans quels cas le bricolage est assumé et quand il est mis « sous silence », l'interaction entre les choix matériels, les enjeux théoriques et le contexte social, l'imbrication du bricolage et du monde professionnel. La notion de bricolage n'était pas au départ une préoccupation centrale dans notre recherche mais elle s'est finalement imposée à travers l'étude des conduites observées sur le «terrain ». La présence du bricolage au sein de toutes les chaînes opératoires qui participent à la fabrication d'images en IRM s'observe chez des professionnels issus de disciplines diverses et investis de statuts différents. Sa présence constante et son étendue confirment son incidence sur l'évolution de la connaissance. Si on se concentre sur la science telle qu'elle se pratique au quotidien dans les laboratoires, nous rencontrons inévitablement des formes d'arrangement étroitement associées aux procédures «standards». Nous observons en fait une articulation profonde entre solutions bricolées et cadre normatif, ce qui rend difficile d'embrasser la séparation 
« classique » entre stratégies et tactiques (de Certeau 1980). Au contraire, nous avançons que dans certaines occasions le scientifique devient bricoleur, non pas pour s'opposer au cadre normatif dominant mais pour mieux répondre à ses impératifs.

Un des aspects originaux de notre approche consiste à poser la question du traitement de l'anomalie dans la recherche scientifique, par le biais du bricolage. Le bricolage intervient parfois comme phénomène intermédiaire entre la règle et l'interdit, et permet de prendre en charge un dysfonctionnement (résultat incongru, difficulté technique, désaccord disciplinaire) tout en assurant le maintien de l'équilibre entre scientificité de la démarche, intérêts personnels, et pressions extérieures.

\section{Bricolages et science}

Une chose bricolée est par définition instable, approximative, fortuite, elle sort de l'ordinaire, s'éloigne des modes opératoires communs. Les usages subjectifs, le caractère « ludique » et le manque de reproductibilité auxquels ces attitudes sont renvoyées expliquent pourquoi, dans le sens commun, bricolage et science se comportent comme des termes antinomiques. Des opérations comme classer, standardiser, prédire, se conjuguent difficilement avec les notions de hasard et d'incertitude.

Le bricolage a été décrit par Levi-Strauss (1962) en opposition à l'ingénierie, en raison de l'absence de projet qui l'anime, de l'importance des moyens matériels disponibles sur le résultat, du caractère aléatoire de la démarche. Si l'ingénieur, se fait guider par un programme préalablement défini lui permettant une mainmise sur le réel, l'activité du bricoleur « dépend » des objets qui l'entourent :

« (...) la règle de son enjeu est de toujours s'arranger avec les "moyens du bord", c'est-àdire un ensemble à chaque instant fini d'outils et de matériaux, hétéroclites au surplus, parce que la composition de l'ensemble n'est pas en rapport avec le projet du moment, ni d'ailleurs avec aucun projet particulier, mais est le résultat contingent de toutes les occasions qui se sont présentées de renouveler ou d'enrichir le stock, ou de l'entretenir avec les résidus de constructions et de destructions antérieures».

L'homme de science, à l'aide de théories et d'hypothèses, « dialogue avec l'univers » alors que le travail du «bricoleur », borné par les événements, se compose d'objets « précontraints ». L'œuvre du « bricoleur » est néanmoins à l'image de ses choix car bien que les limites de son action soient inscrites dans les moyens mobilisés, il témoigne d'une démarche personnelle et unique.

En ouverture de leur ouvrage, Thuderoz et Odin (2010 : 16-17) fournissent une description idéale du bricoleur afin d'en étudier les écarts. Selon leurs représentations, le bricoleur « en transformant un objet, [...] se transforme lui-même ; il est ce qu'il fait ; et ce qu'il fait est noble, puisque non asservi à un objectif de rentabilité. [...] En travaillant pour soi, à son rythme, sans rechercher un quelconque rendement, le bricoleur est tout entier dans le processus [...] N'ayant d'autre maître que lui-même, son travail l'épanouit [...] ». Au contraire l'ingénieur « se met à distance corporelle de son projet ; ce dernier ne le définit pas ».

Pour Derrida (1967) l'idée de l'ingénieur libre des contraintes et exempt de toute forme d'arrangement avec la réalité ne serait qu'un mythe à ébranler. Cette critique apparaît fondée 
quand on a accès aux espaces de production des connaissances comme les laboratoires et qu'on s'intéresse à la science « en train de se faire ». Depuis que l'anthropologie symétrique nous incite à voir en l'hybridité de la science une nécessité épistémologique, le caractère hétéroclite des « faits » scientifiques, investi d'enjeux à la fois humains et non humains, sociaux et techniques, a servi d'arrière-plan à de nombreuses analyses sociologiques (Houdart 2007). Le mélange, le désordre, l'instabilité, ne sont plus des exceptions mais des indicateurs essentiels des stratégies mises en œuvre par le scientifique. Le bricolage perd son acception négative pour devenir une propriété même de la connaissance :

« Les faits scientifiques sont toujours impurs par nature, voire par destination. Ils sont faits de bric et de broc ; ils sont composés d'éléments hétérogènes, associant des compétences à des équipements, des textes à des savoirs tacites, des humains à des non humains. C'est de cette impureté que dépend leur capacité à résister et à intéresser ». (Callon 1989) La dichotomie Levi-straussienne entre bricoleur et ingénieur a été repensée par les sociologues des sciences (Knorr-Cetina 1981, Pickering 1984, Fujimura 1987, Clarke 1990, Kranakis 1990, Lynch 1993) qui ont fourni des descriptions éloquentes quant à la conformation des modes d'action des professionnels à des logiques d'opportunisme et d'adaptation. L'observation du travail scientifique au quotidien révèle le recours à des pratiques de bricolage qui participent activement à la configuration des objets de recherche, des techniques expérimentales, des formes d'écriture.

\section{Bricolage : un concept polysémique?}

Souvent, les inventions scientifiques témoignent d'alliances originales entre des composants matériels disparates ou d'associations d'idées issues de champs épistémologiques différents. Une certaine vision occidentale du savant et du « génie » repose sur cette capacité à combiner des idées appartenant à des univers de significations différents pour aboutir à une solution inédite.

Mais Knorr-Cetina (1981) et d'autres sociologues des sciences ont aussi montré que faire appel au type de raisonnement dit « analogique » sous-jacent au processus de création scientifique n'est pas suffisant pour expliquer les choix effectués dans un contexte donné et qu'il est nécessaire d'invoquer d'autres facteurs, de type environnemental. L'acheminement logique qui accompagne l'élaboration d'une métaphore est orienté par le milieu socio-technique à partir duquel l'idée prend vie : ses objectifs, ses contraintes et ses règles jouent un rôle essentiel. La valorisation d'une solution innovante suit des stratégies qui prennent en compte le contexte dans lequel elle va se développer mais aussi ses retombées : les possibilités techniques, la recherche de collaborateurs, la mobilisation de financement, l'attractivité de l'étude, etc. L'idée est conçue, adaptée aux instruments, rendue stable, communiquée et c'est dans cette chaîne que les mérites sont distribués et que la question de l'origine d'un « fait » scientifique se brouille :

« Les bricoleurs sont des opportunistes. Ils sont conscients des possibilités matérielles qu'ils rencontrent à un endroit donné, et ils les exploitent pour réaliser leurs projets. Dans le même temps, ils reconnaissent ce qui est faisable, et ajustent ou développent leurs projets en conséquence $» .^{1}$ 
La notion de faisabilité, qui a un rôle central dans la définition de l'innovation proposée par Knorr-Cetina, a été reprise par Fujimura pour analyser, dans une ethnographie désormais célèbre (1987 : 261), l'orientation de la recherche sur le cancer au sein d'un laboratoire aux États-Unis. La faisabilité en science pour Fujimura se définit par un agencement "bricolé" des trois espaces du travail scientifique : le cadre expérimental, le laboratoire, le contexte social :

« Je soutiens que les scientifiques parviennent à un alignement en "bricolant" avec ces trois niveaux d'organisation du travail ».

Dans cette acception, la faisabilité dépend étroitement de la réussite de l'articulation entre trois niveaux, celui de l'expérience, du laboratoire, de la société et des ressources et contraintes disponibles:

«Bricoler est un autre terme pour articulation. J'utilise le terme pour sa connotation visuelle et pratique, afin de souligner la construction dynamique des problèmes scientifiques dans des contextes particuliers $»$. (ibid : 261)

Ici, le bricolage n'engage plus seulement une combinaison gagnante d'idées mais une négociation stratégique entre les acteurs disparates d'un réseau.

Successivement le bricolage a été utilisé dans l'analyse de pratiques ordinaires pour faire émerger la dimension « artisanale » du travail scientifique. Morgan Jouvenet (2007) observe notamment des pratiques chez les physiciens, dans le domaine des nanosciences, qu'il range sous le terme de bricolage instrumental. Il s'agit d'un ensemble d'astuces permettant aux acteurs d'accroître l'efficacité des dispositifs techniques, d'améliorer l'environnement de travail, de rendre une expérience plus performante. Jouvenet mentionne, par exemple, les pièces en carton ou aluminium rajoutées sur certains instruments pour amortir le bruit environnant et maintenir la stabilité thermique.

« [S'] ajoutant à cet artisanat de la protection, d'autres menues modifications matérielles visent l'amélioration du confort d'utilisation : c'est le cas des morceaux de scotch qui indiquent la position d'un échantillon sur un écran de contrôle, ou de l'échelle qui y est dessinée au marqueur ».

Il aborde ensuite l'hybridation d'instruments différents à laquelle les physiciens du laboratoire se livrent pour la réalisation de "manips" « originales». Ces deux types de bricolage peuvent être inscrits dans la classification des catachrèses ${ }^{2}$ proposées par Allamel-Raffin sur la base de ses observations en physique des matériaux. Selon elle, il y aurait trois manières possibles de « bricoler » : 1) les catachrèses courantes : « un certain nombre de "trucs" pratiques qui facilitent la vie des chercheurs » (Allamel-Raffin 2005). On peut faire référence ici au détournement d'objets de la vie quotidienne pour combler un manque ou pour remplacer des objets techniques en vue d'en améliorer la performance (comme l'utilisation d'aluminium pour recouvrir certains instruments décrits plus haut);2) les catachrèses conduisant à la création d'un nouvel instrument. Il s'agit de la modification d'un instrument aux fins de lui donner un nouveau pouvoir ; enfin, 3) les catachrèses théoriques qui interviennent « lorsqu'un chercheur recourt à des données théoriques appartenant à un champ disciplinaire différent du sien, et les applique à son propre domaine » (ibid : 31).

Nous croyons que de cette catégorisation émerge une distinction entre la dimension théorique et le monde matériel qui, ainsi que nous l'avons avancé plus haut, ne peut être acceptée a priori. Lassociation analogique entre deux objets ou idées ne peut pas être saisie seulement en tant que raisonnement abstrait. Elle dépend, la plupart du temps, des possibilités matérielles et sociales qui l'accompagnent. Le scientifique est conduit par un ensemble d'interprétations sur la bonne manière d'agir qui est guidée à son tour par le contexte matériel : 
«Les sélections du processus de recherche reflètent des interprétations qui sont des cristallisations de l'ordre dans un espace contingent local ». (Knorr-Cetina)

En s'appuyant sur cette considération et à partir des analyses du bricolage que l'on vient d'esquisser, deux approches se profilent : d'une part, celle de Fujimura et de KnorrCetina, qui appréhende le bricolage comme moyen d'ajustement des niveaux organisationnels différents, d'autre part, celle d'Allamel-Raffin et de Jouvenet, qui se réfère plus particulièrement à des conduites qui facilitent le travail quotidien.

\section{Bricolages et neuro-imagerie}

Dans une première perspective, le bricolage est utilisé pour illustrer la manière selon laquelle le scientifique concrétise une idée qui nécessite un processus d'adéquation des objectifs en vue des possibilités instrumentales, financières, de leur désirabilité sociale et politique, des stratégies de carrière, etc. Comme le souligne Knorr-Cetina innover ne signifie pas seulement suivre des stratégies mentales particulières mais aussi prendre en compte des raisons contextuelles. Ici le bricolage comporte une sélection d'idées qui répondent le mieux aux attentes issues d'espaces sociaux et techniques différents. En retour, le cadre expérimental agit sur l'orientation des programmes de recherche et affecte l'intégration des autres niveaux : le laboratoire et le contexte social. La faisabilité de Fujimura, qui se rapproche de la théorie de l'acteur-réseau de Callon (1989) et Latour (2006) subit les aléas de la « paillasse » et ceux des acteurs institutionnels. Elle est sans cesse renégociée, jusqu'à ce qu'elle fasse l'objet d'un consensus. Ce type de bricolage nous semble être porté vers la réussite d'un fait scientifique, à travers une négociation des équilibres entre objectifs de recherche, alliances politiques et ressources matérielles.

Dans la deuxième approche, le bricolage se réfère aux pratiques qui interviennent plutôt dans des objectifs de réussite à court terme. Ce type de bricolage se rapporte plutôt à des stratégies individuelles contingentes tentant de «faire au mieux avec ce qu'on a à disposition » sans que celles-là pèsent sur l'équilibre entre les niveaux institutionnels ou sans que ce dernier soit le mobile les justifiant. Si, bien évidemment, les deux façons d'appréhender le bricolage ont une incidence réciproque, ils ne coïncident pas toujours. En fait, compte tenu de l'influence indéniable du contexte économique et social sur la manière de circonscrire un problème et d'élaborer des solutions, il y a une faisabilité qui ne découle pas toujours de l'articulation dont parle Fujimura. Le bricolage se structure plutôt autour des aléas techniques et des activités routinières des acteurs sans pour autant mettre en jeu l'articulation des différents niveaux organisationnels.

On va considérer le premier type de bricolage, comme un outil conceptuel employé pour expliquer comment les scientifiques se déplacent d'un objectif à l'autre, ajustent leurs méthodes, improvisent des coopérations, manipulent leurs données en vue de s'adapter aux circonstances matérielles, théoriques, aux stratégies individuelles et au contexte macro-social.

Le deuxième type de bricolage, qui peut être pensé comme une sous-catégorie du premier, peut être revendiqué plus ouvertement par les acteurs car il est ancré dans leurs professions et valorisé par celles-ci. C'est par exemple le cas des physiciens étudiés par Jouvenet qui exposent fièrement l'hybridation instrumentale comme le produit 


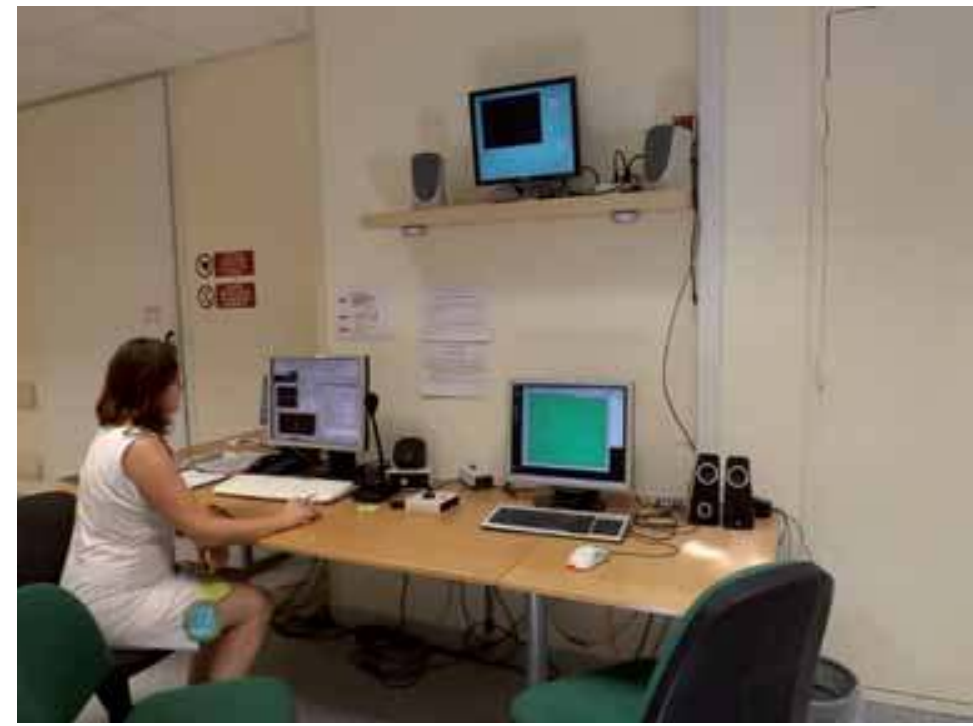

Espaces de travail du centre IRM d'un raisonnement astucieux démultipliant le pouvoir des dispositifs standards, et qui précisent : «Y a qu'ici que tu vois ça... ». Malgré cette valorisation informelle de la débrouillardise, elle est en fin de compte écartée des communications officielles, écrites ou orales. Ce cadrage théorique posé, je rapporterai à présent deux exemples issus de mon terrain d'enquête ethnographique. Ils permettent d'illustrer les deux types de bricolage. L'identification de ces deux catégories n'a pas suivi le même processus; le premier type de bricolage est issu de la reconnaissance de cette pratique par les acteurs eux-mêmes, dans une perspective qu'on pourrait appeler émique. Pour le deuxième type, le terme «bricolage » nous semblait pertinent pour qualifier certaines activités bien que les acteurs n'aient pas mobilisé ce mot pour les désigner.

Notre ethnographie porte sur la cartographie du cerveau et sur l'imagerie cérébrale in vivo. Elle se déroule dans un centre IRM consacré à la recherche en neurosciences cognitives, lieu où les images du cerveau sont produites, traitées, échangées. Le centre IRM se situe dans une institution hospitalière qui abrite un public hétérogène composé de chercheurs provenant de laboratoires spécialisés en neurosciences et psychologie cognitive. Il est géré par trois ingénieurs de recherche qui se livrent à un grand nombre d'activités : formation, recherche de financement, suivi des projets, traitement des images, entretien de la machine, conception de l'instrumentation pour les expériences, etc.

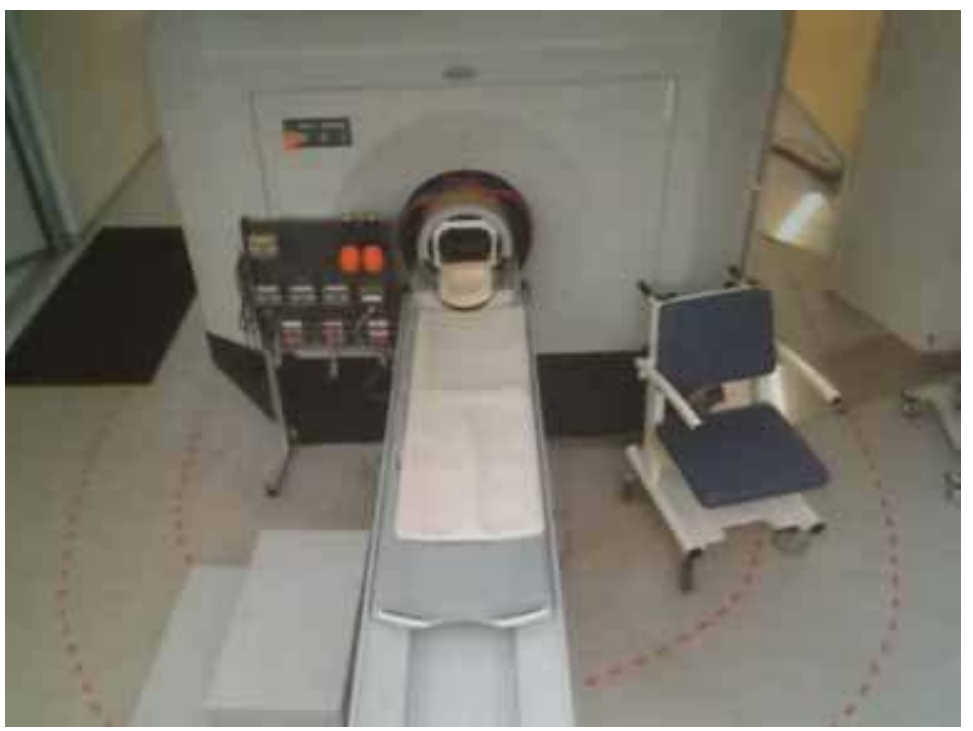

Le quotidien du centre permet de suivre en entier le cycle de vie des images: de la conception à la publication en passant par la production. Avant et après une expérience, les ingénieurs sont présents pour accompagner les chercheurs et les aider à développer leurs questionnements, traiter les données, leur donner du sens en vue de la publication. Durant toutes ces étapes, le scepticisme, les incertitudes et l'imprévu cherchent à être maîtrisés avec des solutions « bricolées » où la négociation et le compromis jouent un rôle essentiel. Le contexte expérimental est particulièrement riche d'enseignements quant à ce type de pratiques. De nombreux facteurs entrent en jeu dans la réussite d'une expérience et les dangers guettent de tous côtés: panne de machine, compétences insuffisantes du chercheur, données récalcitrantes, matériel inapproprié. 
Compte tenu du fait que ces scénarios participent au déroulement normal d'une étude en IRM, les chercheurs et ingénieurs agissent en hommes et femmes rusés, intégrant à leur travail des astuces qui leur permettent de s'acquitter d'une tâche, d'exploiter des données, de combler des lacunes:

« L'intelligence rusée se trouve dans une situation où l'on ne connaît pas la totalité des événements et des forces qui peuvent surgir. L'opération est engagée à travers les ambivalences qui caractérisent cette situation ; elle doit se modifier, changer de parcours en employant tous les trucs dont dispose celui qui les effectue ». (Kanelopoulos 2010 ; Détienne \& Vernant 1974)

Chacun dans sa fonction mobilise le bricolage pour accorder la machine, les images, les sujets des expériences à un projet qui est à son tour reconfiguré par les objets et les acteurs. Les deux exemples suivants fournissent un aperçu de la diversité des fonctions attribuées au bricolage dans l'univers de la neuro-imagerie.

Dans le premier cas, un ingénieur de recherche du centre, fabrique des instruments à partir de matériaux composites alors que dans le deuxième, un chercheur doit agencer les données d'une "manip" IRM pour en faire un récit convaincant et publiable.

\section{Bricoler des objets}

B. est obligé d'intégrer au travail d'ingénieur des capacités de technicien ${ }^{3}$ qu'il mobilise pour concevoir des instruments (simples, comme des boîtiers de réponse, ou plus complexes, comme des tablettes tactiles ou des microphones) utilisés dans les expériences en IRM. Ces derniers doivent à la fois se conformer aux requêtes des chercheurs et être compatibles avec l'IRM, c'est-à-dire être composés de matériaux non ferromagnétiques. Cette contrainte limite le champ d'action de B. et l'oblige à se servir d'objets hétérogènes, parfois issus du quotidien. Ce travail implique, soit la fabrication ex novo, soit la modification d'instruments scientifiques déjà existants. La création d'instruments intègre la récupération d'objets « profanes » ou spécialisés (informatique, électrique, mécanique), l'application de savoirs informels et de connaissances scientifiques.

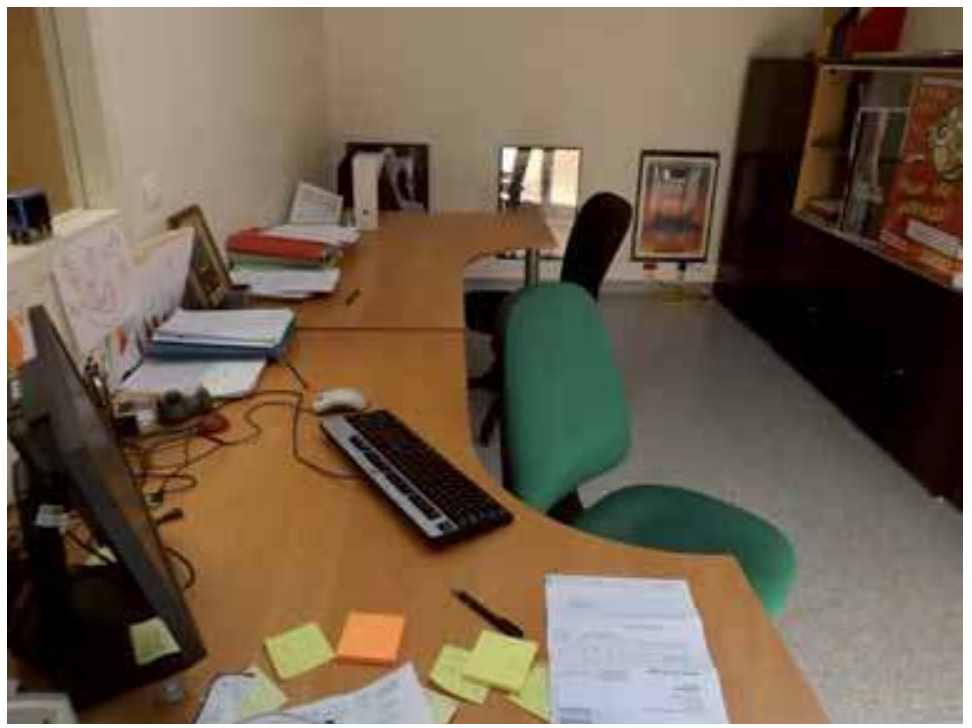


Cet aspect «bricoleur » de son travail est mis en avant comme une spécificité en comparaison d'autres plateformes techniques similaires et lui donne une « valeur ajoutée ». Il faut préciser que la spécialité professionnelle de B., celle de l'ingénieur, valorise les capacités d'adaptation à l'environnement technique et l'élaboration de solutions « arrangées » mais performantes, une véritable mètis.

Le bricolage fait partie des atouts de l'ingénieur et cette habilité supplémentaire constitue une valeur ajoutée appréciée par les chercheurs et les membres du centre. Nous allons montrer brièvement quelques exemples d'objets techniques « bricolés » par B. pour rendre compte des gestes et des savoirs mobilisés, d'une part, et des fonctions de ces pratiques de bricolage, d'autre part.

\section{Oxymètre}

L'oxymètre, qui mesure l'oxygénation du sang, est utilisé pour surveiller, à l'aide d'un moniteur, un singe lors d'une expérience en salle IRM. Le vétérinaire a besoin de savoir si, durant l'acquisition des images, le cœur du singe, qui est préalablement anesthésié, bat à une vitesse régulière. Le dispositif informe sur les battements cardiaques grâce à un capteur placé sur la pointe d'un doigt de la main. À l'usage, B. s'aperçoit que le capteur ne se fixe pas correctement sur le doigt et il décide d'en fabriquer un autre: « le système d'attache était pourri, il y avait des scratchs qui ne tenaient pas... ». Pour fabriquer ce capteur, B. a ici utilisé une pince à linge en plastique qui a été d'abord privée de l'élément métallique (le ressort) et qui a ensuite subi des modifications pour qu'elle soit adaptée à la tâche. B. a percé l'épingle en deux endroits afin d'appliquer l'émetteur et le récepteur, il a bloqué les deux fils avec du scotch et a fixé à l'aide d'un élastique les deux composantes de l'objet.

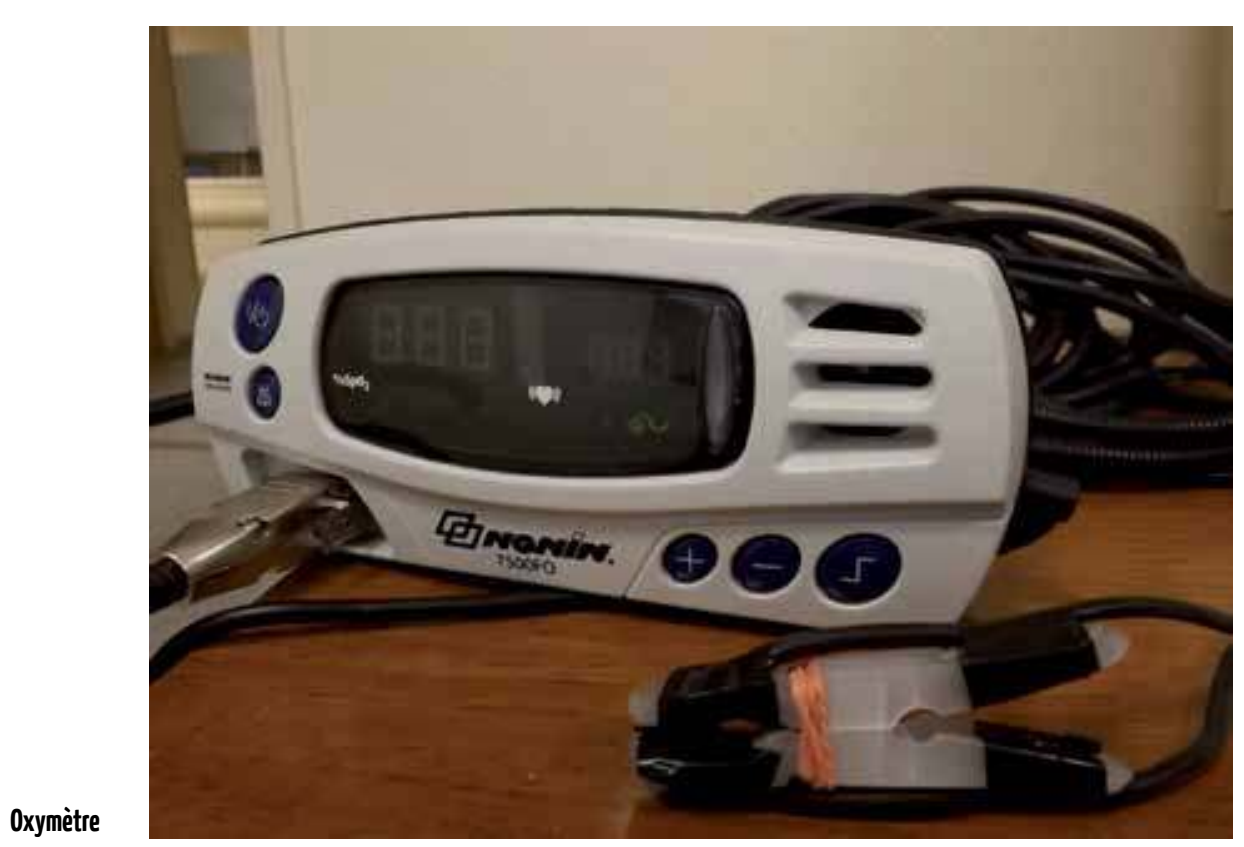




\section{Boutons et boîtiers de réponse}

Objet classique de la psychologie expérimentale, les boutons de réponse par lequel le sujet communique avec l'expérimentateur, doivent ici s'adapter aux contraintes techniques de l'IRM et être entièrement constitués de matériaux non ferromagnétiques. Pour la fabrication de ces objets B. réutilise des flacons d'aspirine dont la taille et la forme se prêtent bien à la prise en main. Son espace domestique n'est pas tout à fait sans influence dans la conception de cet objet, la femme de B. étant pharmacienne. Outre leur ergonomie, ces objets ont l'avantage d'être en plastique et de posséder une cavité dans laquelle on peut insérer les fils électriques. B. perce alors les flacons, applique de petits boutons de couleurs différentes et fixe les fils avec des soudures. Parfois, pour obtenir une meilleure adhérence de la main, il recouvre les flacons de guidoline, gaine antidérapante fixée aux cintres des bicyclettes.

Il existe des dispositifs de réponse similaires mais plus complexes dont nous avons suivi la fabrication. B. est sollicité pour s'occuper de l'instrumentation nécessaire à des mesures expérimentales lors d'une opération de chirurgie cérébrale d'un patient. Le but est de stimuler certaines zones du cortex à l'aide d'électrodes et ensuite d'enregistrer les réactions du sujet. On fait appel à B. pour synchroniser un premier ordinateur qui recueille les réponses comportementales et un deuxième qui reçoit les activités électriques cérébrales. Les réactions du sujet aux stimuli sont communiquées à travers un boîtier de réponse qui doit également être fourni aux chercheurs et dont nous allons retracer la fabrication.

En premier lieu, B. prend un boîtier en plastique qu'il ouvre et perce là où il veut faire passer les fils électriques et colle deux boutons sur sa partie extérieure. Il « câble » ensuite les boutons, reliant deux fils électriques par bouton à l'aide de soudures en étain. Il assemble les parties du boîtier et le referme soit avec des vis, soit, s'il est destiné à des expériences IRM, avec du scotch.

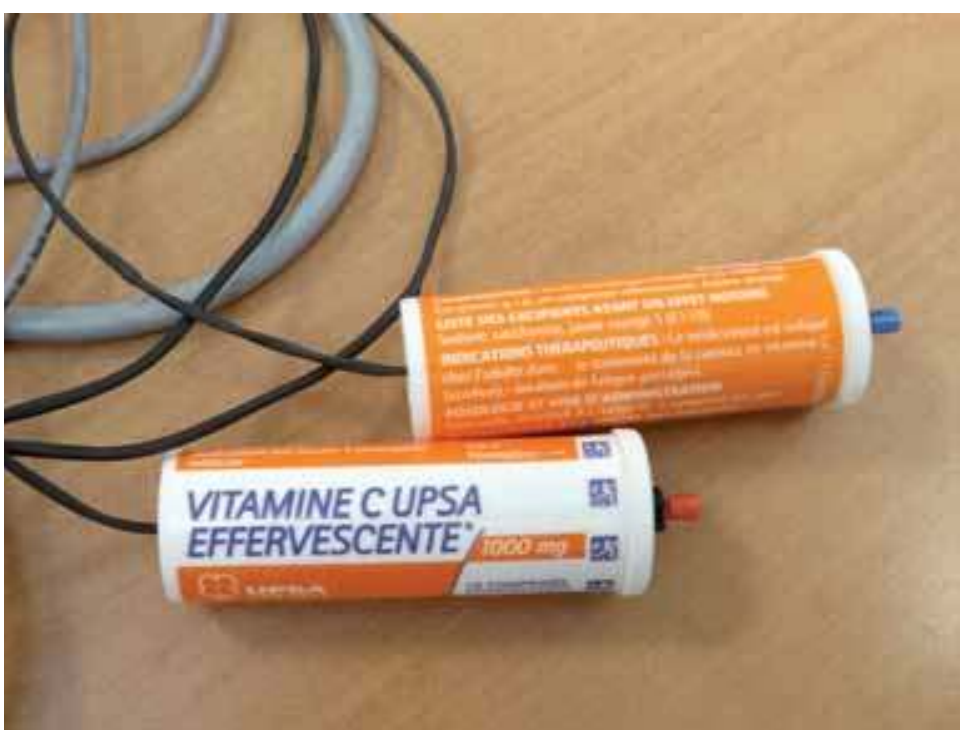

Boutons de réponse avec et sans gaine

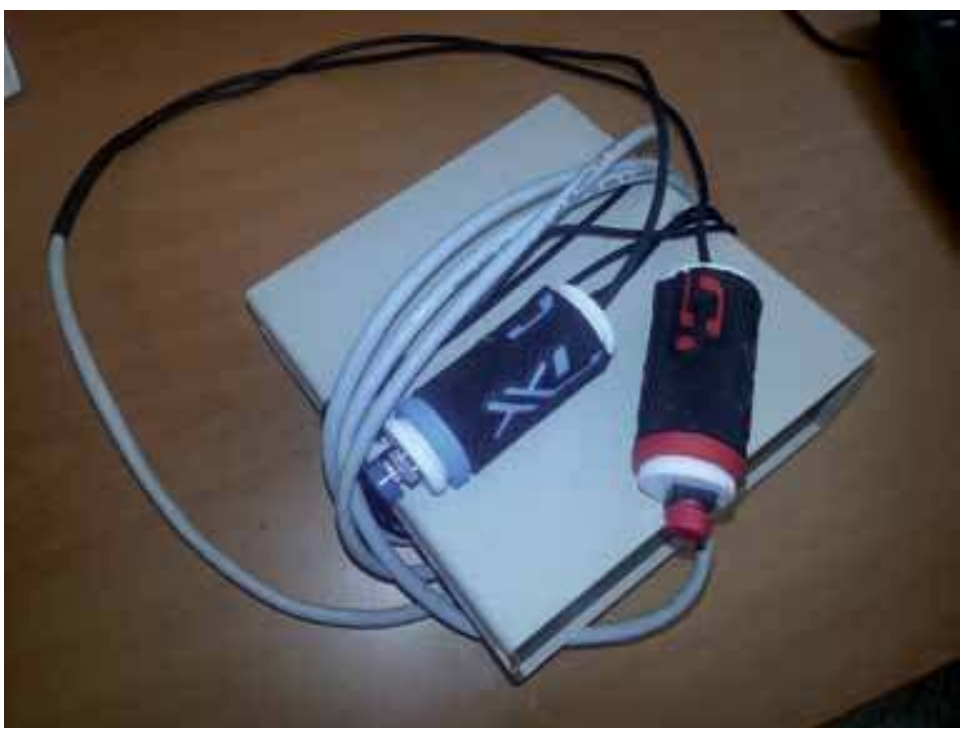




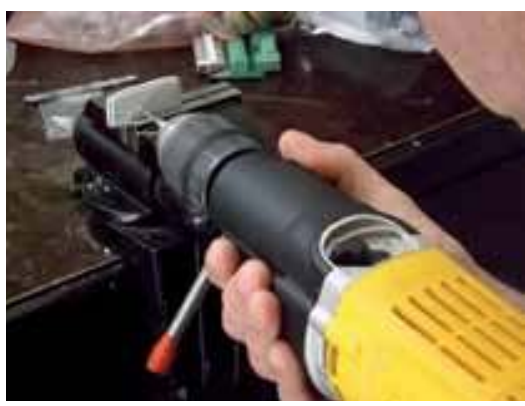

\section{Fabrication d'un boittier \\ de réponse}

1. Perçage d'une partie

du boîtier

2. Application de la colle

3. Fixation des boutons

4. Réalisation de la soudure
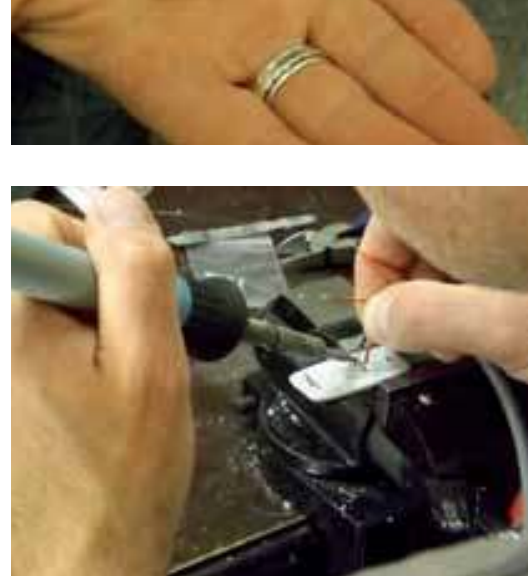

5. Mise en place des fils

6. Jonction des fils aux

boutons
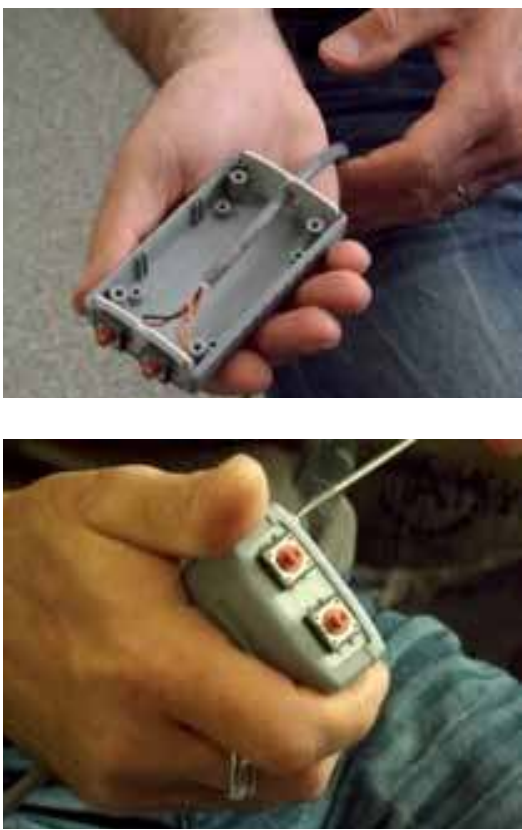

9. Fermeture avec vis

10. Fermeture avec scotch
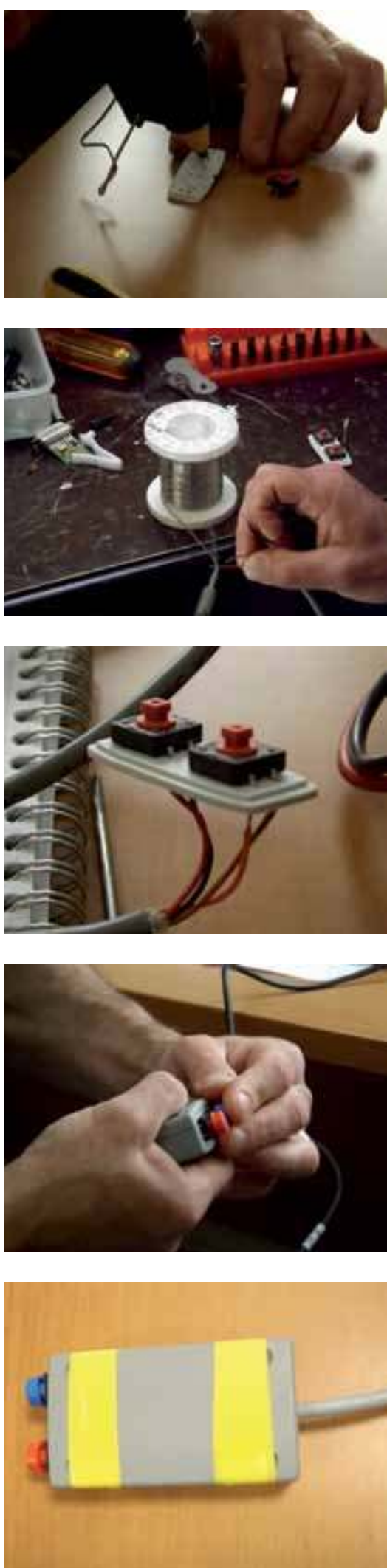


\section{Webcam compatible IRM}

Les difficultés rencontrées par les expérimentateurs pour suivre de près l'état de santé du singe alors qu'il se trouve dans la machine ont amené $\mathrm{B}$. à concevoir une webcam mobile qui puisse être placée dans la salle IRM afin d'observer l'animal en temps réel. L'hétérogénéité des matériaux utilisés saute aux yeux lorsqu'on regarde le résultat de l'assemblage : bois, plastique, aluminium, plaques électroniques. Le support qui donne leur stabilité aux autres parties est un porte-cravates que B. a trouvé dans le bureau. Le tube articulé en plastique bleu (qui est d'habitude un tuyau pour lubrifiant intégré à des machines-outils) a été découvert par B. grâce à un ami mécanicien qui lui en a suggéré l'idée. Cet objet anodin pour le mécanicien, a trouvé son potentiel auprès de l'ingénieur qui l'a aussitôt acquis via un site Internet spécialisé, et utilisé à ses fins. La webcam prévue pour des ordinateurs portables a été achetée dans une grande surface. Privée de la plaque en fer qui permet de l'accrocher à l'écran de l'ordinateur, elle est enveloppée d'un film d'aluminium suivant les principes de la « cage de Faraday » qui isole le dispositif des ondes électromagnétiques.

Même si la fabrication de ces objets est rarement nécessaire parce que ceux-ci sont souvent disponibles à la vente et que $\mathrm{B}$. «n'est pas tenu de faire ça », il préfère se charger personnellement de la conception et de l'assemblage de ces produits pour plusieurs raisons :

«Dans le commerce tu peux en trouver mais ça coûte un prix démesuré et l'avantage de le construire c'est que tu le construis comme tu veux, tu l'interfaces comme tu veux, si jamais il y a un truc qui tombe en panne tu sais le réparer et surtout c'est toi qui choisis la taille, les composants, la couleur...».

Bricoler est alors un bon moyen pour parer aux limites des produits manufacturés, au coût élevé mais aussi afin de maîtriser les connaissances. La rapidité de B. permet de contenir les temps de fabrication, beaucoup plus avantageux

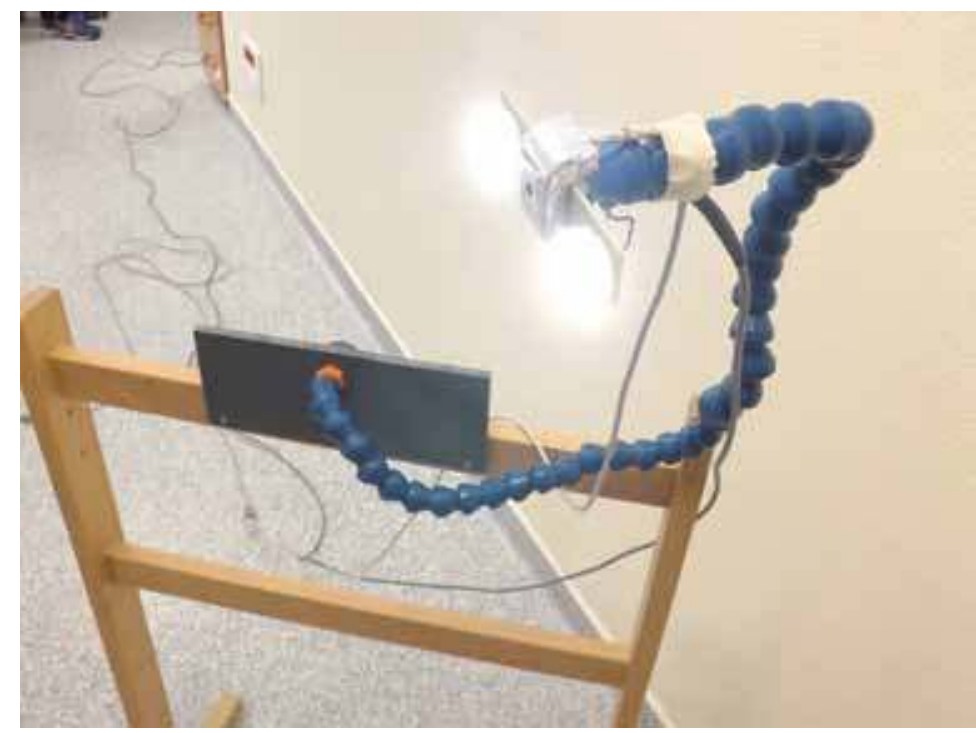

Webcam de surveillance

d'un « PNH » (« primate non humain ») en salle IRM

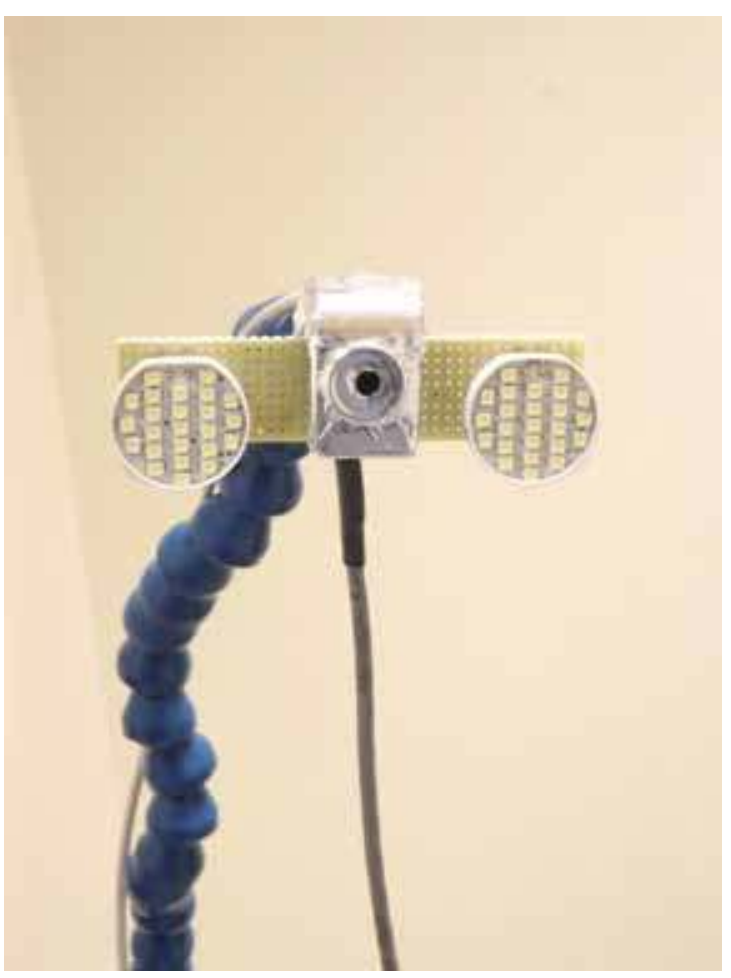



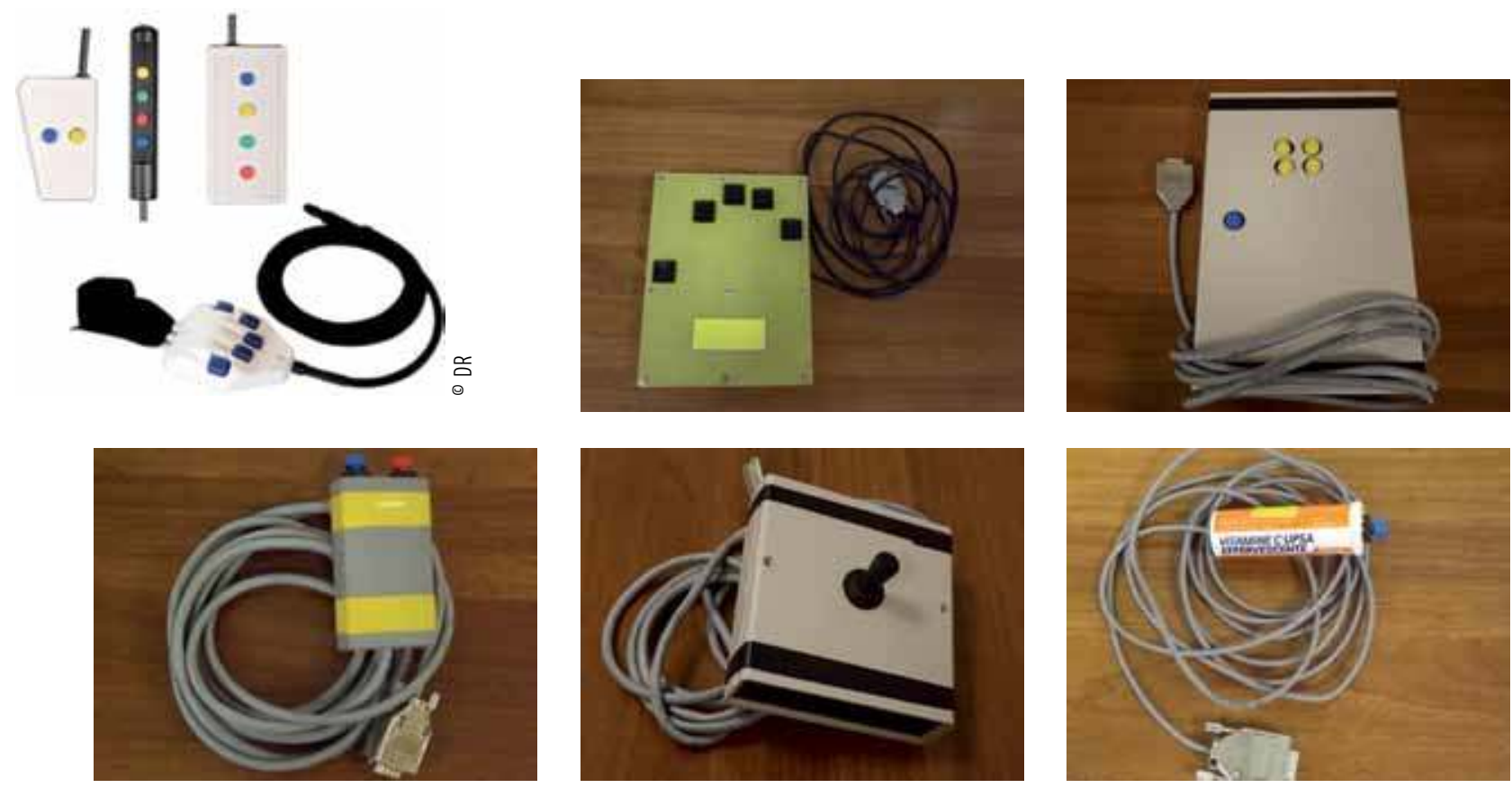

Claviers et boutons de réponse commercialisés (en haut à gauche) et bricolés par B. (à droite, ci-dessus) en comparaison des délais d'attente d'une commande sur le web ; le boîtier de réponse est, par exemple, achevé en moins d'une demi-heure. En outre, ces moments permettent à B. d'alterner travail informatique et activité manuelle ; opérations techniques et choix créatifs se combinent.

Incorporer des objets anodins à des dispositifs techniques complexes en fait des objets originaux et amène à faire du neuf avec des bribes de matériel en fin de vie (boutons de clavier d'ordinateur, flacons d'aspirine...). B. conçoit les dispositifs à partir des exigences avancées par chaque chercheur. Des inventions prennent vie tout en restant souterraines, car leur usage est réservé aux expérimentations du centre. La conception d'un clavier de réponse avec quatre boutons rapprochés que l'on voit ci-dessus a été mise au point pour s'adapter à la position des stimuli tels qu'ils étaient présentés au sujet lors d'une expérience. Adapter l'objet au mode d'affichage des stimuli amène à la production d'un objet unique, non pas dans son genre mais dans les fonctions qu'il assure. Mais les mêmes procédés, caractérisés par l'articulation d'idées et de matériaux hétérogènes destinés à la résolution d'un problème ou d'un besoin contingent, peuvent aussi occasionner une innovation. Je ne pense pas seulement aux objets mais aussi à des programmes informatiques de traitement d'images qui sont « bricolés » et combinent des fonctionnalités différentes puisées dans des logiciels concurrents pour en augmenter la puissance. Ces expériences peuvent parvenir à la création de nouveaux outils qui sont ensuite mis à la disposition de la communauté des neuro-imageurs.

Si les produits du bricolage de B. doivent faire l'objet d'une publication, ils seront définis à partir de leurs propriétés générales. Pour désigner la webcam par exemple on fera référence à un dispositif de vidéosurveillance sans que cela nécessite d'explications supplémentaires. Dans ce cas, l'occultation du bricolage n'opère pas par dissimulation d'informations, mais par camouflage, c'est-à-dire que pour parler de dispositifs bricolés dans les communications officielles on se servira d'une terminologie codifiée partagée. 


\section{Bricoler des images}

Les résultats issus des expériences scientifiques ne «sortent » pas tels quels du laboratoire ; ils doivent répondre à des exigences théoriques, techniques, littéraires pour être rendus publics. Respecter les conventions qui régissent le travail de rédaction et attribuer aux observations empiriques une valeur théorique donnée implique une valorisation de certains aspects de la recherche et un oubli volontaire de certains autres. Cette succession de sélections est souvent difficilement discernable comme l'a déjà constaté Knorr-Cetina :

« Larticle scientifique cache bien plus que ce que sa façade apprivoisée et civilisée laisse transparaître. Premièrement, il oublie délibérément une grande partie de ce qui s'est passé dans le laboratoire, bien qu'il vise à présenter un « rapport » de cette recherche. Deuxièmement, les résultats écrits d'une recherche usent pour une bonne part de stratégie littéraire qui reste largement peu perceptible aux lecteurs ». (ibid: 94) ${ }^{4}$

Dans le cas suivant, les données issues d'une expérience en neurosciences ne donnant pas satisfaction (en termes de significativité statistique), ont dû être « réadaptées » afin de faire l'objet d'une démonstration destinée à être publiée dans un article. De plus ces données, produites quelques années auparavant lors d'une expérience en IRM dans le cadre d'une thèse de doctorat et n'ayant pas fait l'objet d'un article, ont été récupérées par un autre chercheur travaillant sur les mêmes aires cognitives qui voulait s'en saisir pour une publication. L'ingénieur de recherche responsable de la salle IRM, précise que : « c'est dommage de ne pas publier les données, même si elles ne sont pas belles... ». En effet, il est rare qu'une expérience IRM ne donne pas lieu à une publication vu l'investissement qu'elle nécessite en matière de temps et d'argent.

La question est alors double : quand on est en présence de données de « deuxième main » que l'on n'a pas produites soi-même et qui ne confortent ni les hypothèses de départ ni les études existantes, comment « se débrouille-t-on » avec tout ça?

J'ai été en possession de deux documents, le premier informel, à destination des collègues, qui expose les données à l'état «brut » et, d'un second, version initiale d'un article. Je tiens à préciser que le premier des deux documents n'est pas une ébauche de l'article modifié par la suite mais qu'il s'agit davantage d'un outil de travail à partir duquel le chercheur échange avec les ingénieurs du centre IRM et son superviseur. Ce document contient un résumé des résultats et des procédés utilisés pour les analyser, mais surtout énonce ouvertement des doutes sur la pertinence des méthodes choisies, et des questions concernant le sens à attribuer aux images, ce qui nous donne un accès privilégié à la façon d'organiser les données pour qu'elles soient recevables par la communauté des chercheurs en imagerie cérébrale.

\section{« Faire avec ce quion a et ce quion náa pas... »}

L'analyse d'images IRM et la visualisation des activations cérébrales sont possibles grâce à l'utilisation de logiciels spécialisés qui permettent de «lire » les données «brutes». Il faut d'abord rendre les images « comparables» en les modifiant pour qu'elles soient placées dans le même espace-temps. Ce processus automatique, le prétraitement, doit être bien maîtrisé pour réussir les analyses statistiques qui suivront. Chaque chercheur a sa " recette de cuisine » et choisit les paramètres 
selon sa propre expérience. Une fois le «batch ${ }^{5}$ approuvé, le chercheur est censé l'appliquer à ses données et travailler sur les images obtenues. Mais dans la pratique les chercheurs procèdent par essai-erreur, c'est-à-dire qu'ils testent plusieurs méthodes et choisissent celle qui leur a donné la plus grande satisfaction en termes d'images. Le texte qui nous a été transmis, permet de saisir cette pratique très commune mais jamais assumée, du moins officiellement. Il s'agit par exemple du choix entre plusieurs types de normalisation, paramètre qui uniformise les cerveaux au niveau spatial ${ }^{6}$. Le chercheur expose les avantages et les défauts de chaque méthode après avoir précisé :

«Ce que je fais d'habitude consiste à prendre l'image anatomique, à la découper, à ajuster la matière grise et blanche segmentée avec les cartes $\mathrm{MNI}^{7}$, et à appliquer ces paramètres aux images EPI ${ }^{8}$. Alors que cela fonctionne généralement assez bien, nous avons un problème avec cet ensemble de données, car la taille des images anatomiques chez certains sujets ne correspond pas complètement à la taille des images fonctionnelles. ${ }^{9}$ ".

La méthode habituelle du chercheur étant inefficace, il essaie plusieurs procédures qui ne lui conviennent pas entièrement. Pour chacune d'elles, il écrit les " pour » et les « contres » et le choix final se fixant sur la méthode qui s'adapte le mieux aux images qu'on lui a transmises:

« Toutefois, je vais présenter ici les résultats réalisés avec l'approche de normalisation "segAnat", parce que la majeure partie de l'analyse s'appuie sur ces données. Et entre la peste et le choléra... ». ${ }^{10}$

La tâche cognitive de l'expérience cherche à faire activer des zones du cerveau traitant les informations «visuelles », la zone traitant le mouvement appelée V5 ou $\mathrm{MT}^{11}$ (middle temporal) est située dans la partie dorsale du cortex et celle consacrée à la couleur, nommée V4, est située dans la partie ventrale. On demande au sujet de cliquer à gauche ou à droite au moyen d'un clavier de réponse selon la couleur des stimuli affichés sur l'écran (ainsi par exemple : bleu = gauche). La tâche est compliquée par le fait que, lors de la présentation, les stimuli bougent à droite et à gauche. Ils sont considérés « compatibles » si le mouvement est dans la même direction que celle associée à la couleur du stimulus (par exemple : pour un stimulus bleu on demande de cliquer à gauche et il bouge vers la gauche) ou « incompatibles » dans le cas où le mouvement est dans la direction contraire à celle associée au stimulus (le stimulus bleu bouge vers la droite). Lactivation de la zone V5 est prédite lors de l'apparition de couples de stimuli dont les premiers sont compatibles alors que la zone V4 devrait s'« allumer » pour des couples de stimuli dont les premiers sont incompatibles. Pour mieux identifier les deux zones, V4 et V5, chez les sujets, l'expérience prévoyait une tâche préliminaire d'observation passive de stimuli très colorés et de stimuli en mouvement. Cette tâche-test, appelée localizer ${ }^{12}$, est rajoutée dans l'espoir que les deux zones « allumées » seront les mêmes que celles impliquées dans la véritable tâche expérimentale.

Mais malheureusement ce ne sera pas le cas et les activations prédites par les hypothèses et localisées par la tâche fonctionnelle ne correspondent pas avec celles activées dans le reste de l'expérience. Quand l'activation de V5 est attendue on observe des zones plus antérieures, et pour V4, en plus d'être placée antérieurement, l'activité est plus faible et moins reconnaissable, ainsi qu'on peut le comprendre dans la figure présentant les résultats de différentes activations cérébrales. 


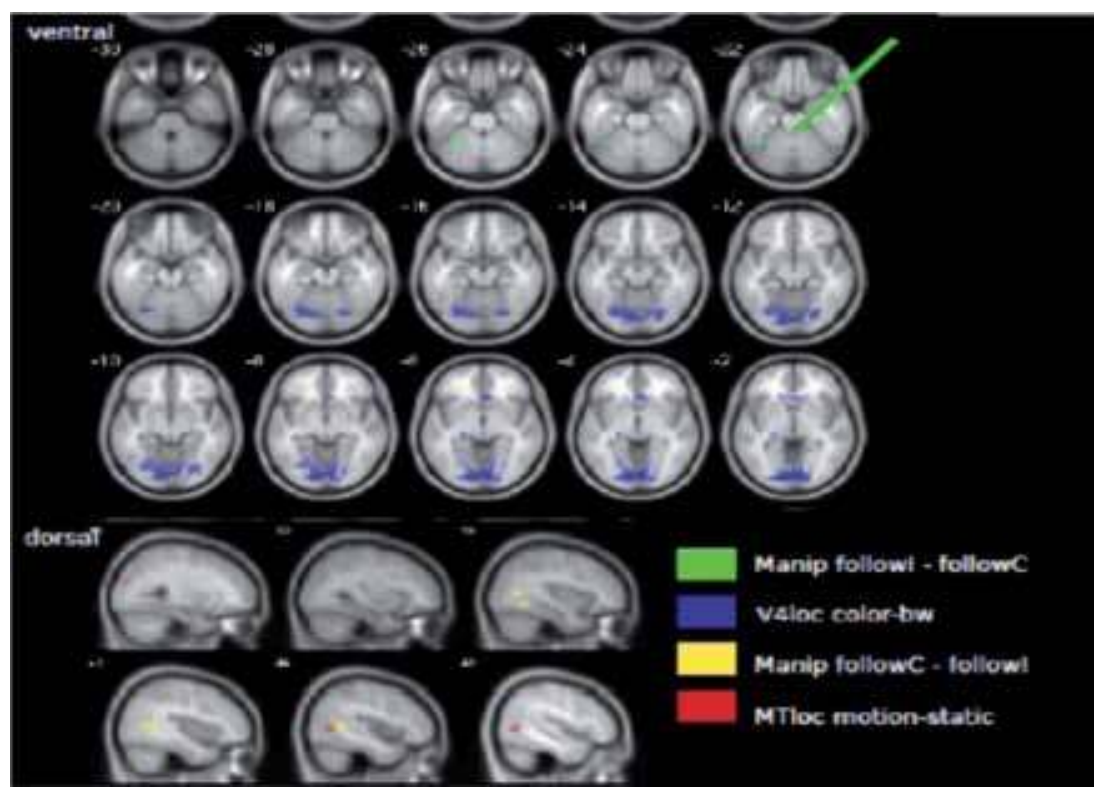

Dans une discussion entre le chercheur et le responsable de la salle IRM, ce dernier conseille alors de ne pas mentionner le localizer dans l'article final pour que l'échec des hypothèses soit moins flagrant. Dissimuler cette opération et les images qui en résultent permet d'élargir le champ des possibles, de ne pas le restreindre à l'avance avec une prédiction forte sur la localisation des zones impliquées dans la tâche.

Il ne faut pas seulement faire une sélection des informations émergeant de l'expérience, mais il faut aussi adopter un mode de présentation des données qui, sans changer leur nature, puisse les présenter sous un jour acceptable par rapport aux attentes du chercheur et aux expériences relatées dans la littérature. Les zones activées sont visualisées dans un espace antérieur à celui qui a été prévu et identifié au départ par le localizer. Ce dernier donc est problématique pour circonscrire les $\mathrm{ROI}^{13}$, les régions d'intérêt, les aires visuelles occipitales V4 et V5. Le chercheur essaie alors plusieurs méthodes pour délimiter des zones dans les voies ventrale et dorsale qui incluent les activations de l'expérience. Cela peut être fait de plusieurs manières : en retraçant une sphère autour d'une région délimitée par des coordonnées anatomiques définies par la littérature, en suivant les activations issues de la "manip", ou encore en s'appuyant sur des zones « allumées » lors du localizer. Mais aucune méthode ne permet d'inclure efficacement les données de l'expérience. Voilà un extrait des tentatives effectuées par le chercheur :

" - Légère variation: Prendre l'ensemble de l'activation visuelle du contraste $\mathrm{rfx} \mathrm{X}^{14}$ pour le groupe et le seuil à une valeur élevée de $\mathrm{T}$; cela ne fonctionne pas pour l'activation dorsale, car il ne fait pas partie du "cluster" [ensemble de "voxels" : contraction de volumetric pixel] mais fonctionne pour l'activation ventrale ;

- Prendre une sphère autour du maximum global du "cluster" obtenu avec la séquence de repérage ; cela ne fonctionne pas pour les activations ventrales et dorsales car elles sont vraiment loin (il faudrait une sphère de plus de $20 \mathrm{~mm}$ ) ;

- Prendre les cartes probabilistes de la boîte à outils pour l'anatomie ; il y a des problèmes avec la normalisation MNI ; si je parviens à normaliser raisonnablement le cortex
Différences entre activations cérébrales attendues et activations cérébrales provoquées

En bleu et en rouge, les zones activées par le localizer; en jaune et vert, les zones activées lors de la tâche. 
occipital (segEPI), l'activation ventrale s'en va, d'ailleurs, les deux activations sont en dehors de ces cartes de probabilité ;

- Prendre le contraste V4 de la séquence de repérage - expérience pour obtenir les régions pertinentes des taches visuelles; ça fonctionne pour l'activation ventrale ; ne fonctionne pas pour l'activation dorsale ; et comment peut-on justifier ça ? ${ }^{15}$ ».

Le chercheur essaie plusieurs modes opératoires mais bien souvent les paramètres qui « marchent » pour visualiser les zones dans la voie ventrale ne fonctionnent pas pour la démarcation des activations dans la voie dorsale. Les possibilités qui apparaissent au chercheur dans l'article final sont doubles : définir la ROI avec la méthode qui fonctionne le mieux ou omettre ces étapes et prendre en compte le cerveau « entier » (wholebrain analysis) sachant que l'échec des prédictions initiales sera manifeste. À la fin, il penche pour cette dernière solution en choisissant le test statistique le plus restrictif parce que : « (...) l'analyse de ROI serait plus "cosmétique" que nécessaire. En outre, notre véritable hypothèse physiologique (V4 et MT) n'était pas vraie ».

Ce constat n'est pas rendu tel quel dans l'article qui met en avant dans l'introduction la conformité des hypothèses initiales qui ont été confirmées par les données (la modulation des activations dans le traitement de la couleur et du mouvement des stimuli dans des essais « incompatibles ») plutôt que la divergence entre la prédiction de localisation et les résultats. Le lecteur sera incité à remarquer l'activation des zones "adjacentes" à V4 et MT.

À ce point de la discussion une distinction importante s'impose. Le bricolage de données que nous venons d'examiner n'est pas assimilable à une fraude qui renverrait, elle, à un « acte malhonnête » qui viserait à tromper d'autres personnes en contrevenant aux règlements et qui engage des punitions (formelles ou informelles). La fraude scientifique, dans sa définition restreinte, se mesure en termes de fabrication ou falsification des données, et de plagiat. Altérer des résultats, avancer des preuves créées de toutes pièces pour appuyer une théorie, revendiquer la propriété de découvertes déjà existantes fait partie des comportements frauduleux sanctionnés par la communauté scientifique. Ici, le chercheur n’agit pas sur la nature des données mais sur la forme seule de leur énonciation. Les choix réalisés dans l'écriture, la dissimulation de certaines informations ne transforment pas l'échec en succès mais rendent la défaite moins rude.

Pour ce qui concerne l'application de plusieurs méthodes aux mêmes données, c'est une pratique très courante, qui, bien qu'elle soit mal perçue dans ce secteur de la science, est malgré tout tolérée. Cela s'explique en partie à cause de l'ancrage de ces conduites aux moyens techniques employés. Les effets produits par chaque paramètre d'un logiciel de traitement sur les images sont variables et parfois inexpliqués, même pour les «spécialistes ». Cela incite les chercheurs à tester différentes manières d'analyser les résultats.

L'ingénieur du centre IRM commente ainsi les opérations résumées par le chercheur dans son texte:

« Moi je lui ai dit t'as même pas le droit de faire ça... il me dit : oui mais on le fait tous! Oui mais t'as pas le droit (...) Parce que si tu prends des données et tu les testes un milliard de fois avec des méthodes différentes tu finiras bien par trouver quelque chose à force, on gratte les données, on les secoue un peu pour voir s'il y a pas quelque chose qui tombe...».

Ce jugement n'entraîne pas de conséquences déontologiques, il s'agit davantage d'un procédé « à risque » que d'une fraude véritable. Il convient donc de séparer les deux phénomènes, la fraude et le bricolage même si nous n'excluons pas que «bricoler » des 
données puisse parfois accompagner des conduites frauduleuses. La fraude et le bricolage peuvent en fait servir le même but, celui de l'emporter dans des milieux compétitifs :

« La fraude, [...] peut être définie hors approche philosophique comme une stratégie évolutive pour la survie dans certains types de situation concurrentielle ». ${ }^{16}$ (List 1985)

Précisément parce qu'il se positionne à la limite entre ce qui est accepté et ce qui est interdit, le bricolage relève d'une occultation plus importante.

Dans le «brouillon » de l'article tous les moments d'incertitudes en rapport à la bonne façon de procéder, les incohérences des résultats, les nombreux tests appliqués aux images disparaissent et un compte rendu linéaire décrit seulement certaines étapes des traitements qui ont été suivies. Les faits sont agencés de façon à composer un portrait cohérent ; même si les données ne le sont pas, et on l'a vu, cela implique une adaptation forte de la méthode aux résultats obtenus.

Face aux éventuels échecs encourus dans l'expérimentation, la réfutation des hypothèses initiales, les désaccords sur les résultats, le scientifique « bricole » pour répondre à des contraintes techniques mais aussi personnelles, sociales et institutionnelles.

\section{À la recherche d’une définition}

Le « bricolage » est une bonne entrée pour aborder la complexité du travail scientifique parce qu'il donne accès à la face « cachée » du quotidien du laboratoire, à savoir l'ensemble des situations d'insatisfaction et d'incertitude, auxquelles il faut réagir au mieux.

À l'aide des réflexions émergeant de la littérature et de nos propres observations ethnographiques, nous avons identifié le bricolage à deux moments du travail scientifique : en amont de la production des expériences et pendant le traitement des données. Le bricolage peut répondre à des objectifs différents, même si souvent ces objectifs se confondent et se recoupent.

Les acteurs peuvent s'emparer du bricolage pour améliorer les performances d'un acte, les qualités ergonomiques d'un objet, la puissance d'une machine, le pouvoir explicatif d'un modèle théorique ou l'efficacité d'une méthode. Ce bricolage comporte l'assemblage d'éléments théoriques et d'objets hétérogènes et s'évalue dans le cadre restreint de la réalisation d'une tâche précise. C'est cette dernière qui pose les limites du champ d'action. Dans ce sens, le bricolage est conçu comme une capacité à dépasser les conventions (par exemple, se servir d'objets du quotidien pour construire des dispositifs techniques) pour répondre de manière originale, parfois innovante à une question de recherche. Les « débris d'événements » (Levi-Strauss 1960), employés par l'ingénieur pour fabriquer ses instruments ne sont pas accusés d'une altération du projet initial, au contraire, ils l'appuient en favorisant son accomplissement.

Dans d'autres situations le scientifique déploie ses efforts pour assurer la "survie » d'un énoncé, d'une expérience et la légitimité de son travail suite à l'évaluation de ses pairs. Les acteurs doivent « bricoler » pour faire face à un problème relatif aux normes socio-techniques qui pèsent sur leur travail. Dans le cas analysé, le chercheur ne transforme pas les images, il leur fait subir plusieurs traitements, dans une démarche exploratoire, pour mieux circonscrire les activations attendues. Avec ces procédés, le chercheur contourne la règle selon laquelle on doit appliquer une seule méthode et s'en 
tenir aux résultats obtenus. La valorisation d'une production des connaissances basée sur l'exploration de données à l'ère du numérique explique pourquoi cette manière de « tester » les données est largement pratiquée et tolérée. Ces comportements pouvant dériver et basculer dans l'« interdit », et se situant à la limite de l'« acceptable », ne sont pas rendus publics ; on n'en laisse guère de trace au moment de la rédaction d'un article. L'occultation est décisive eu égard aux « risques » encourus par le scientifique s'il affichait publiquement ce type de pratique.

Ces deux types de bricolage apparaissent à des temps différents dans la production du savoir scientifique : le premier entraîne l'adaptation des moyens à la tâche, le deuxième implique l'ajustement des résultats à l'objectif, et vice versa, de l'objectif aux résultats. La scientificité semble moins compromise dans le premier type de bricolage et son occultation, est donc moins importante. Elle s'opère par camouflage plutôt que par omission.

Nous avons défendu un lien profond entre bricolage et monde scientifique. Jouvenet fait correspondre le bricolage aux tactiques de de Certeau, en relation à ses observations sur le terrain des nanotechnologies. Pour lui :

« Dans les laboratoires, les initiatives "tacticiennes" s'appuient sur une culture professionnelle valorisant les capacités anti-routinières de création et d'improvisation du chercheur, et concurrencent les stratégies (hiérarchiquement) descendantes dans la définition des orientations thématiques de l'organisation scientifique ». (ibid : 200)

Mais nos exemples démontrent que le bricolage ne s'oppose pas au cadre normatif dominant. Dans l'exemple où les données sont ajustées aux contraintes d'une publication scientifique, si les procédures n'incarnent pas la norme, c'est finalement pour y répondre qu'un bricolage s'accomplit. Les fonctions stratégiques de ce type de pratiques expliquent pourquoi la rentabilité, la compétition et les règles qui dirigent la production du savoir scientifique, non seulement ne sont pas contradictoires avec le bricolage, mais le légitiment pleinement. Le bricolage devient alors une arme pour l'affirmation de soi, l'acceptation par la communauté scientifique, l'adaptation à un milieu technique.

\section{$\&$}

Le bricolage, s'est, en définitive, révélé un concept opérant pour résumer la gestion des moments « critiques » qui minent la réalisation d'une expérience de recherche. Dans le cas de la production d'images IRM, une dissonance entre les observations empiriques et les prédictions peut amener le chercheur en neurosciences à trouver des solutions « hybrides ». La publication des résultats atteste en fait du compromis entre ce que le chercheur « voit » et ce qu'il veut « montrer ». Le statut ambigu du bricolage de données conduit le chercheur à faire « disparaître » les traces qui relèveraient d'une affiliation à ces pratiques.

Malgré l'existence de types de bricolage différents nous pouvons toutefois tracer une définition à l'aide de trois principes qui leur sont communs : le détournement, c'est le cas de l'utilisation d'objets techniques à des fins différant de leur fonction initiale. Cela peut impliquer aussi la récupération et la migration de modèles théoriques, savoirfaire, méthodes d'un domaine disciplinaire à un autre ; l'hétérogénéité : cette propriété est visible par exemple dans le mélange de matériaux et connaissances scientifiques et 
non-scientifiques pour fabriquer des objets ou améliorer des instruments ; l'ajustement : qui implique la création de quelque chose à partir de ce que l'on a à disposition. Ces trois principes touchent aussi la question de la faisabilité : le champ des possibles se définit et se redéfinit pour s'adapter aux circonstances.

Dans cet article nous avons avancé deux acceptions du bricolage pour pouvoir penser un vaste champ de pratiques engagées au quotidien par le scientifique en réponse à des problèmes contingents. Pour dépasser ces derniers on peut « bricoler » pour maximiser l'efficacité d'opérations techniques ou théoriques, ou bien pour réduire la portée d'un échec ou bien encore désamorcer une menace à l'ordre constitué. Loin d'être exhaustives, ces catégories sont susceptibles d'être affinées mais nous permettent maintenant non seulement de tracer des frontières et d'avancer des comparaisons dans nos ethnographies respectives mais aussi de réfléchir sur notre propre travail de chercheur en sciences humaines. 


\section{NOTES}

Photo d'ouverture: Superpositions bricolées. Puzzle apposé sur la gravure d'un cerveau colorisé (@) CC0 1.0 \& G. Anichini) Toutes les illustrations sont de l'auteur sauf mention contraire.

1. «Tinkerers are opportunists. They are aware of the material opportunities they encounter at a given place, and they exploit them to achieve their projects. At the same time, they recognise what is feasible, and adjust or develop their projects accordingly » Knorr-Cetina, K. $1981: 34$.

2. Suivant la définition du CNTRL (Centre national de ressources textuelles et lexicales) une catachrèse est un « procédé qui étend l'emploi d'un terme au-delà de ce que permet son sens strict ». Cette notion linguistique est employée par l'auteur comme synonyme de bricolage.

3. La différenciation entre technicien et ingénieur de recherche est avancée par $B$. pour faire valoir sa fonction première qui est celle d'assister les chercheurs, avec les autres ingénieurs du centre, dans l'utilisation de la machine et la mise au point des techniques nécessaires à la réalisation d'une expérience. Cela dépasse pour lui le rôle du «simple technicien».

4. «The scientific paper hides more than it tells on its tame and civilised surface. For one thing, it deliberately forgets much of what happened in the laboratory, although it purports to present a «report» of that research. Second, the written products of research employ a good deal of literary strategy largely unnoticed by the readers. » Knorr-Cetina, ibid : 94

5. Liste d'instructions qui seront réalisées automatiquement par l'ordinateur.

6. Pour pouvoir rendre « comparables» des images de cerveaux de taille et de forme différentes il faut les ramener à un espace commun, après avoir produit une superposition des images anatomiques et des images fonctionnelles.

7. Montreal Neurological Institute.

8. Echo Planar Imaging, images fonctionnelles.

9. «What I usually do is to take the anatomical image, segment it, normalize the segmented grey $\&$ white matter to MNI maps, and apply those parameters to the EPI images. While it usually works pretty good, we have a problem in this dataset because the size of the anatomical images in some subjects does not fit the size of the functional images completely ».

10. «I will however here present the results done with the segAnat normalization approach, because most of the analysis relies on those data. And between pest \& cholera...».

11. Plus récemment un distinguo a été introduit : hMT (human Middle Temporal) chez les humains et MT (Middle Temporal) chez les singes.

12. Séquence de repérage.

13. Region Of Interest.

14. Random effect analysis (analyse de variance à effets aléatoires).

15. «- Slight variation: Take the entire visual activation of the "all" rfx contrast for the group and threshold at a high T value; does not work for dorsal activation because it is not part of the cluster; works for ventral activation - Take a sphere around the global maximum of the localizer experiment cluster; does not work for ventral and dorsal activations because they are really far off (one would need a sphere of more than 20mm) - Take the probabilistic maps of the anatomy toolbox; problems with MNI normalization; if I manage to reasonably normalize the occipital cortex (segEPI) the ventral activation goes away; besides that, both activations are outside of these probability maps - Take the contrast V4localizer ("all") - experiment ("all") to get the visual task-relevant regions; works for ventral activation (see Fig 9); does not work for dorsal activation; And how would one justify this? »

16. «Fraud, (...) may be defined in nonethical terms as an evolving strategy for survival in certain kinds of competitive situation ». List, C. J. 1985 : 28. 


\section{RÉFÉRENCES}

Akrich, M. 1987 Comment décrire les objets techniques ?, Techniques \& Culture 9 : 49-64.

Allamel-Raffin, C. 2005 Instruments et bricolage en physique des matériaux : l'exemple des catachrèses, Tracés 9.

Bloor, D. 1983 Socio-logie de la logique ou les limites de l'épistémologie, traduit de l'anglais par Dominique Ebnöther, Paris : Pandore (Collection Pandore).

Callon, M. (dir.) 1989 La Science et ses réseaux. Paris : La Découverte - Conseil de l’Europe, Unesco.

Clarke, A., Fujimura, J. (dir.) 1996 La Matérialité des sciences : Savoir-faire et instruments dans les sciences de la vie. Paris: Synthélabo Groupe.

Certeau, M. de 1980 L'invention du quotidien. 1, Arts de faire. Paris : Gallimard (Folio Essais).

Derrida, J. 1967 L'Écriture et la différence. Paris : Seuil.

Détienne, M., Vernant, J.-P. 1974 Les Ruses de l'intelligence. La mètis des Grecs. Paris : Flammarion.

Fujimura, J. 1987 Constructing Doable Problems in Cancer Research: Articulating Alignment, Social Studies of Science $17:$ 257-293.

Houdart, S. 2007 La Cour des miracles. Ethnologie d’un laboratoire japonais. Paris : CNRS Éditions.

Hutchins, E. 1995 Cognition in the Wild. Cambridge/London : MIT Press.

— 1995 How a cockpit remembers its speeds? Cognitive Science 1995/19 : 265-288.

Jouvenet, M. 2007 La Culture du «bricolage » instrumental et l'organisation du travail scientifique. Enquête dans un centre de recherche en nanosciences, Revue d'anthropologie des connaissances 2007/2 (1) 2 .

Kanelopoulos, C. 2010 Travail et technique chez les Grecs. L'approche de J.-P. Vernant, dans Anthologie raisonnée de Techniques \& Culture, Techniques \& Culture 54-55 2010/1-2. Paris : Éditions de la Maison des sciences de l'homme : 335-353.

Knorr-Cetina, K. 1981 The Manufacture of Knowledge. An Essay on the Constructivist and contextual Nature of Science. Oxford : Pergamon Press.

Kranakis, E. 1992 Hybrid Careers and the Interaction of Science and Technology. In Peter Kroes and Martijn Bakker (dir.) Technological Development and Science in the Industrial Age, Boston Studies in the Philosophy of Science 144, Dordrecht : Kluwer : 177-204.

— 1990 Technology, Industry, and Scientific Development, in Solomon's House Revisited : The Organization and Institutionalization of Science. Tore Frängsmyr (dir.) Nobel Symposium 75. Canton, MA : Science History Publications : 133-159.

Latour, B. 2006 Nous n'avons jamais été modernes. Essai d'anthropologie symétrique. Paris : La Découverte (Poche / Sciences humaines et sociales).

Lemonnier, P. 2012 Mundane Objects: Materiality and Non-verbal Communication. Walnut Creek (US) : Left Coast Press.

Levi-Strauss, E. 1962 La Pensée sauvage. Paris : Plon.

List, C. J. 1985 Scientific Fraud: Social Deviance or the Failure of Virtue?, Science, Technology, \& Human Values (10) 4 (Autumn 1985) : 27-36.

Lynch, M. 1993 Scientific Practice and Ordinary Action: Ethnomethodology and Social Studies of Science. New York : Cambridge University Press.

Odin, F., Thuderoz, C. (dir.) 2010 Des mondes bricolés? Arts et sciences à l'épreuve de la notion de bricolage. Lausanne : PPUR (METIS LyonTech). 
Pickering, A. 1984 Constructing Quarks: A Sociological History of Particle Physics. Chicago : University of Chicago Press.

Schon, D. A. 1963 Displacement of Concepts. London : Tavistock.

Vinck, D. 1999 Ingénieurs au quotidien. Ethnographie de l'activité de conception et d’innovation, Grenoble : PUG.

\section{POUR CITER CET ARTICLE}

Anichini, G. 2014 Quand c'est la science qui bricole, c'est du sérieux, in S. Boulay \& M.-L. Gélard, Vivre le sable! Corps, matière et sociétés, Techniques \& Culture 61 : 212-235. 


\section{RÉSUMÉ}

Quand c'est la science qui bricole, c'est du sérieux. Loin d'être une pratique accessoire à la production de la connaissance scientifique, le bricolage affecte tous les niveaux du travail quotidien du chercheur, de l'ingénieur, du technicien. L'analyse des observations relatées par les sociologues des sciences et celle des données issues d'une enquête personnelle réalisée au sein d'un centre IRM (Imagerie par Résonance Magnétique) ont abouti à la reconnaissance de deux types de bricolage qui ont été établis sur la base de leur impact sur le produit final, d'après le type de contrainte qui les justifie et selon les techniques d'occultation auxquelles les acteurs ont fait appel pour les dissimuler.

\section{ABSTRACT}

When science does odd jobs, it's serious. Rather than being a subsidiary practice for the development of scientific knowledge, tinkering affects work dynamics in researchers, engineers and technicians everyday life. On the basis of the analysis of results provided by sociologists of science and following observations from a field work realised in a MRI (Magnetic Resonance Imaging) lab, we will identify two categories describing the practice of tinkering. To establish this categorisation we will take into account the impact of the bricolage on final products, the type of constraints that justify it and the concealment techniques recruited to minimize its consequences.

\section{MOTS CLÉS}

Bricolage, production des connaissances, ajustement, technologie, IRM, anthropologie des sciences

\section{KEYWORDS}

Bricolage, knowledge production, technology, adjustment, MRI, anthropology of sciences 